BNL -64637

\title{
The New BNL Partial Wave Analysis Programs *
}

\author{
John P. Cummings Dennis P. Weygand
}

July 29,1997

\section{Introduction}

\author{
:
}

Experiment E852 at Brookhaven National Laboratory is a meson spectroscopy experiment which took data at the Multi-Particle Spectrometer facility of the Alternating Gradient Syncrotron. Upgrades to the spectrometer's data acquisition and trigger electronics allowed over $\mathbf{9 0 0}$ million data events, of numerous topologies, to be recorded to tape in 1995 running alone. One of the primary goals of E852 is identification of states beyond the quark model, i.e., states with gluonic degrees of freedom. Identification of such states involves the measurement of a systems spin-parity.

Such a measurement is usually done using Partial Wave Analysis. Programs to perform such analyses exist, in fact, one was written at BNL and used in previous experiments by some of our group. This program, however, was optimized for a particular final state, and modification to allow analysis of the broad range of final states in E852 would have been difficult. We therefore decided to write a new program, with an eye towards generality that would allow analysis of a large class of reactions.

\section{Partial Wave Analysis Formalism}

\subsection{General Description of Pwa}

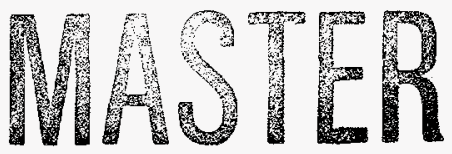

Partial Wave Analysis, or PWA, is a technique used in hadron spectroscopy to extract information about the spin-parity and decay properties of resonances produced in hadronic interactions. Typically, these resonances are produced at accelerator experiments via a variety of production mechanisms. The resonances produced by these methods also appear in the decay products of other well known resonances, such as the $J / \psi$, and can be studied there. Although the tools we describe here could be used in an investigation that uses any of these production mechanisms, perhaps with slight modification, so far they have been used only in peripheral production experiments, and so we will use this type of production as an illustrative example in this paper.

The general idea is to parameterize the intensity distribution in terms of variables that have physical meaning when interpreted as properties of interme-

1

*Work performed under the auspices of U.S.D.O.E., Contract \#DE-ACO2-76CHOOO16. 


\section{DISCLAMEER}

Portions of this document may be illegible in electronic image products. Images are produced from the best available original document. 


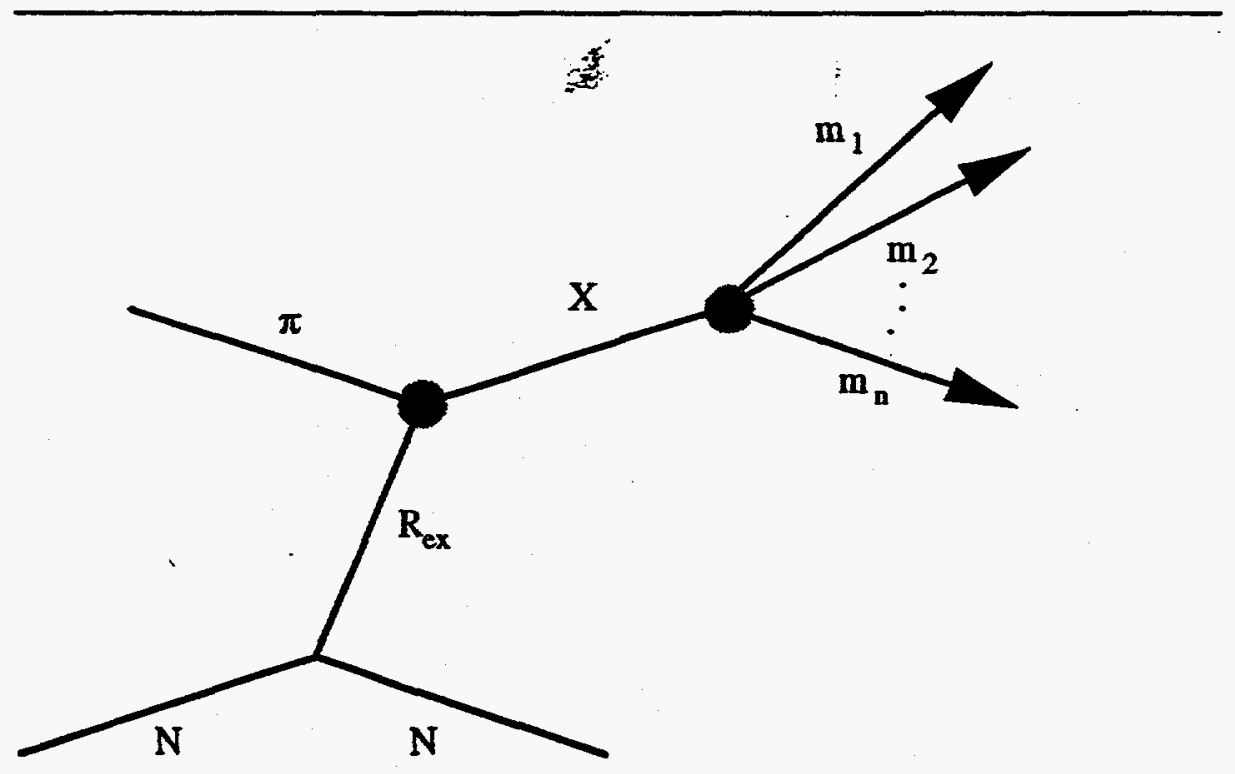

Figure 1: Diagram representing the amplitude $\psi_{\alpha \beta}(\tau)$. The intermediate state $X$ is produced by the exchange of a Reggion $R_{\text {ex }}$ between the $\pi$ beam and the nucleon target.

’ 


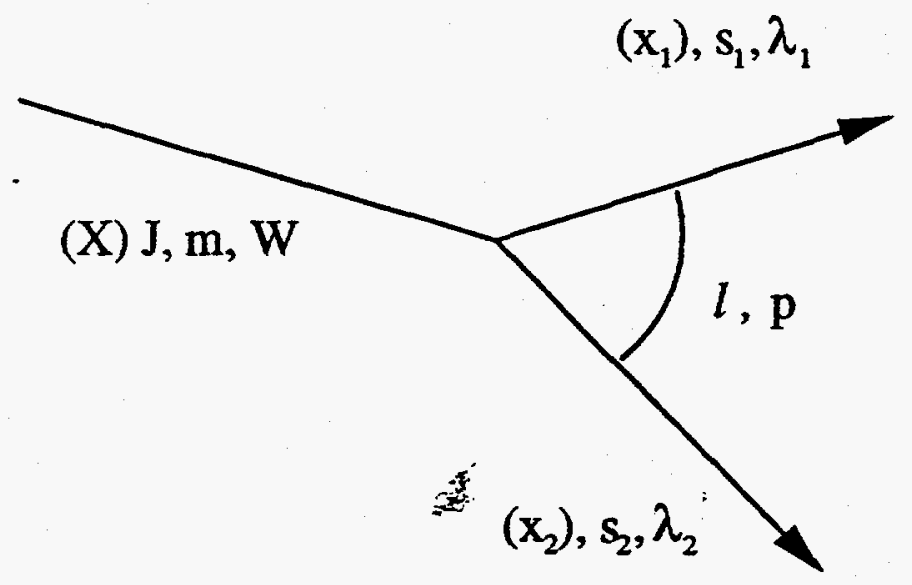

Figure 2: Decay of the resonance $X$ into $x_{1}$ and $x_{2}$.

The initial decay of the $X$ into its children is evaluated in the GottfriedJackson frame. This frame is a rest frame of the resonance $X$ with the $z$ axis in the direction of the beam and the $y$ axis perpendicular to the production plane. The quantities used in defining the amplitude are shown schematically in Figure 2 The state $X$ of mass $W$ has spin $J$ with $z$ projection $m$. It decays into children $x_{i}$ with spin $\sigma_{i}$ and helicity $\lambda_{i}$. These children have a breakup momentum $p$ and relative orbital angular momentum $l$. The decay amplitude can then be written down [Chu71]

$$
A_{X}=\check{l} \sum_{\lambda} D_{m \lambda}^{J *}(\Omega)(l 0 s \lambda \mid J \lambda)\left(s_{1} \lambda_{1} s_{2}-\lambda_{2} \mid s \lambda\right) F_{l}(p) a_{l s} A_{z_{1}} A_{x_{2}}
$$

where $\lambda=\lambda_{1}-\lambda_{2}$ and $\vec{s}=\vec{s}_{1}+\vec{s}_{2}$, i.e., $\vec{s}$ is the total spin of the two children and $\lambda$ is the component of $\vec{s}$ in the direction defined by $x_{i}$ 's momentum. The $A_{x_{i}}$ are the decay amplitudes of each child.

The $\bar{\ell} \equiv(2 \ell+1)^{1 / 2}$ factor, along with the two Clebsch-Gordan coefficients, come from the fact that we are using helicity states and must relate the helicity coupling constant to the $\ell s$ coupling constant $a_{\ell s}$ we want to find through the partial wave expansion. [Chu71, section 4.3]

Rotational properties of the helicity states lead to the $D$ function $D_{m \lambda}^{J_{*}}(\Omega)$ where $\Omega=(\theta, \phi, 0)$ are the Euler angles of $x_{1}$ in the Gottfried-Jacisson frame. The choice of the third angle $\gamma=0$ defines a phase convention different from that of Jacob and Wick [JW59], who choose $\gamma=-\phi$.

$F_{\ell}(p)$ is an angular momentum barrier factor added to give the amplitude the correct behavior near threshold. We used the Blatt-Weisskopf centrifugalbarrier functions as given by von Hippel and Quigg. [vHQ72]

Finally, the $a_{\ell s}$ is the $\ell s$ coupling constant which contains the dynamics of 
tivistic Breit-Wigner, although it is sometimes necessary to use a more complex parameterization, such as coupled-channel Breit-Wigners [Fla76] or a $K$-matrix parameterization [CK95].

Total decay amplitudes are made up as a product of these intermediate decays. In order to parameterize the spin-density matrix, which is determined by the fit, in the simplest way, it turns out to be advantageous to transform the states into the reflectivity basis. The reflectivity basis is defined by eigenstates of reflection in the production plane, the details of the transformation can be found in Chung and Trueman. [CT75]

\section{Program Description}

The BNL partial wave analysis programs were designed using object-oriented methods to produce a general system for doing partial wave analysis. The resulting objects were implemented using $\mathrm{C}++$. Four programs comprise the basis of the system, although numerous support programs and scripts have been developed.

\section{1 gamp}

The program gamp (generate amplitudes) is the decay amplitude calculator. The command line useage is available by running the program with no arguments:

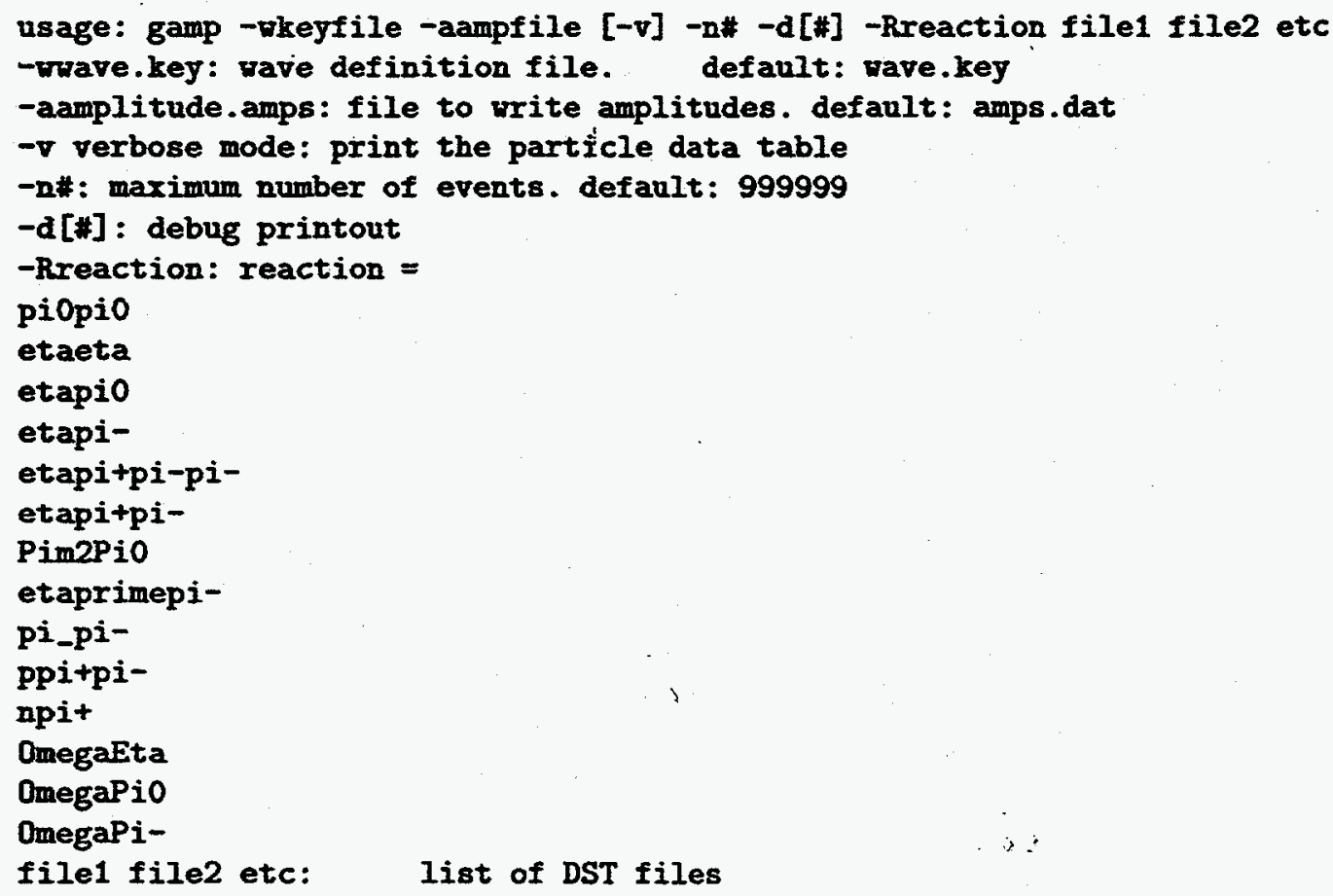


up to the second ":" the decay of the $X$ is given in the format isobar/bachelor, $l$ where $l$ is the orbital angular momentum of the isobar decay. Had this reaction had additional isobar decays, they would be specified in an analogous way between the second and third ":"'s, etc.

The datafile, at present, must be in an E852 itape format containing an event summary record (ESR) group. For purposes of analyzing other data, for example LASS data described below, custom conversion programs were written to put the data into the required format.

\section{2 int}

The program int calculates the normalization integrals used in the maximum likelihood fit. It also has command like usage available:

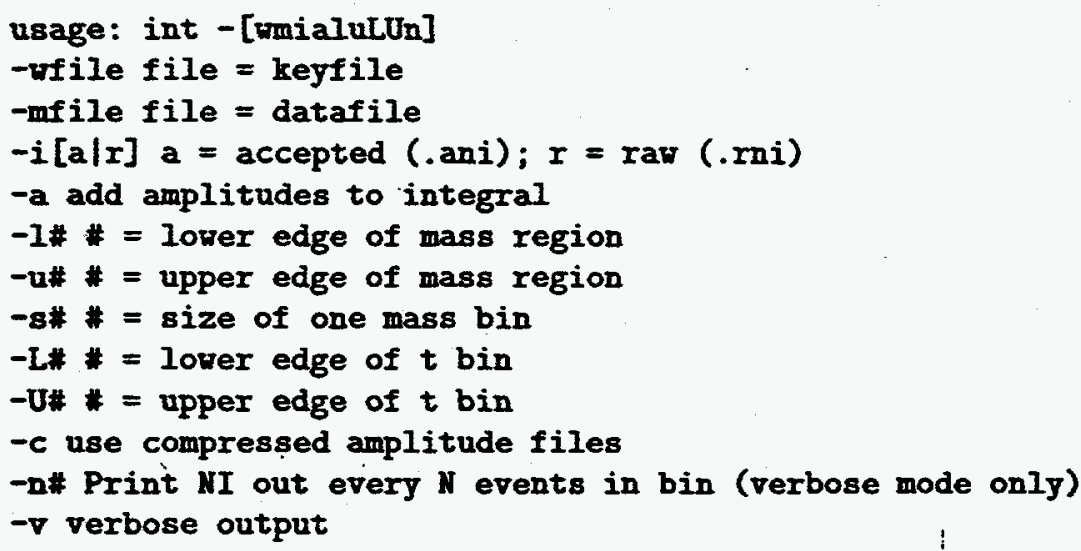

It needs as input a keyfile and amplitudes for all waves in the keyfile for the Monte-Carlo datafile. This is a simple program that just reads amplitudes from disk and performs the sum in equation 4, writing as output a matrix of complex numbers to nifiles. The indices of the matrix correspond to the waves in the keyfile. This links a set of normalization integrals to a particular keyfile which must be used in the fit. To say this another way, if the set of waves used in the fit is changed, then the normalization integrals must be recalculated.

The normalization integrals are done in mass and momentum transfer bins that correspond to the bins that will be used in the fit. The values of the ranges for these values, as well as the keyfile that was used is encoded into the names of the nifiles. For example, the file names

Kset13_2:1.32-1.36:0.00-0.15: .ani

Kset13_2:1.32-1.36:0.00-0.15:.rni

Kset13_2:1.32-1.36:0.10-1.00: .ani

Kset13_2:1.32-1.36:0.10-1.00:.rni

Kset 13_2:1.32-1.36:0.15-0.30: . ani

Kset13_2:1.32-1.36:0.15-0.30:.rni 
successive fit commands for a range of bins typically takes care of the initial generation of MINUIT parameters before running fit.

For each bin in which the script runs fit, an output file is generated containing the fit results. These logfiles contain all the information available about the fit results: fitted production amplitudes, normalization integrals, the keyfile, and the error matrix from the fit. Any observable may be calculated from this.

\section{4 xvf}

$x \nabla f^{1}$ is a windowing post-processor program which uses the logfiles produced by fit to calculate observables. The appearance of the program is shown in Figure 4. The fit being looked at is a fit of the $\eta \pi$ final state data recorded in the 1994 run of Brookhaven experiment 852. Plotted is the number of events, acceptance corrected, that are assocfatted with the partial waves selected in the left pane. In this example, the $2^{++}$amplitudes are selected. Both are amplitudes are for $D$-wave decays into $\rho^{0} \pi^{-}$, one having $m=0$ and the other having $m=1$.

$x \vee f$ calculates this predicted number of events using

$$
N=\frac{n}{\eta_{x}} \sum_{\alpha \alpha^{\prime}} V_{\alpha} V_{\alpha}^{*} \Psi_{\alpha \alpha^{\prime}}
$$

The normalization integral $\Psi_{\alpha \alpha^{\prime}}$ is calculated using int, and $\frac{n}{\eta_{\alpha}}$ is the number of observed events in a bin divided by the acceptance. The $V_{\alpha}$ are the production amplitudes found by the fit. In the sum over waves, $\alpha$ takes on all values selected, such as all the $2^{++}$waves above.

Ivf can also display other observables using similar formulae, such as the phase difference between two production amplitudes or the magnitude (abs) of the production amplitudes.

\subsection{Support Programs}

In addition the four major programs in the suite, there are also various support programs that are used in an analysis. wr $2 \mathrm{~min}$, for example, reads a keyfile and produces a list of fit parameters in a format that is understood by MINUIT. This program is generally run from a script such as dofit. dofit is written in Perl and takes care of running wormin, running fit in a range of bins specified, possibly using the results of a previous bin to initialize the next fit. Similar scripts exist to run int, and, as mentioned above, gamp.

ViewAmps is a very simple program which is used extensively for diagnostics. It simply reads an ampfile and writes the mass, $t$ and amplitudes to standard output.

\footnotetext{
${ }^{1}$ xvf was written by Alexander Ostrovidov based on a much cruder program written by one of this papers authors.
} 


\section{Internals}

In this section we give a brief description of the internal workings of the programs and the design philosophy that was used. We begin with a description of the general strategy adopted to calculate the decay amplitudes in a very flexible way, allowing extension to many final states. An outline of the objects developed to accomplish this is then given.

\subsection{General Strategy}

The isobar model leads naturally to the idea of a recursive algorithm to calculate the total decay amplitude. Each level of the recursion calculates the contribution to the total due to individual two body decays in the sequence.

\subsection{Description of Objects}

\subsubsection{Matrices, etc.}

Essential to any particle physics analysis is the notion of vectors (both three and four dimensional) and matrices of an arbitrary dimension. We choose to write a threeVector class first, and a fourVector contains a threeVector (We felt this was a more natural implementation than using inheritance). All the immediately obvious operators and functions were defined for the vectors: addition, subtraction, scalar multiplication, vector multiplication, rotations and boosts (using the Matrix class), transformations from a cartesian to a polar representation, etc.

A Matrix class provided for arbitrary size (and shape) matrices of real numbers, and once again, most of the typical algebraic operators are supplied. In addition, a CMatrix class proved to be very useful for partial wave analysis, where it is often convenient to write the (complex) amplitudes using a matrix notation. It is maybe is interesting to note that while this would be a textbook example of a use for templates, we wrote these classes before the introduction of templates into the $\mathrm{C}++$ language. The CHatrix class was written (almost) completely by the UNIX commands:

sed 's/double/complex/' Matrix.hxx > Clatrix.hxx

sed 's/double/complex/' Matrix.cxx > CMatrix.cxx

\subsubsection{Waves and Amplitudes}

Partial waves are specified in the code using the WaveType class. WaveType has public data members for the quantum numbers of the system being studied and its decay products, which are stored as arrays of ParticleData:

class WaveType \{

public:

int $J$;

int $P$ :

$/ *$ Total spin $* /$
$/ *$ Intrinsic parity */ 
[CK95] Su Urk Chung and Eberhard Klempt. A primer on $K$-matrix formalism. BNL Preprint Uni Mainz IP 92-03, Brookhaven National Laboratory, March 1995. Version VII.

[CT75] S. U. Chung and T. L. Trueman. Positivity conditions on the spin den- sity matrix: a simple parametrization. Physical Review D, 11(3):633646, February 1975.

[Fla76] S. M. Flatte. Coupled-channel analysis of the $\pi \eta$ and $K \bar{K}$ systems near $K \bar{K}$ threshold. Physics Letters, 63B:224, 1976.

[JW59] M. Jacob and G. C. Wick. On the general theory of collisions for particles with spin. Arnals of Physics, 7:404-428, 1959.

[vHQ72] Frank von Hippel and C. Quigg. Centrifugal-barrier effects in resonance partial decay widths, shapes, and production amplitudes. Physical Review D, 5(3):624-638, February 1972.

\section{DISClAIMER}

This report was prepared as an account of work sponsored by an agency of the United States Government. Neither the United States Government nor any agency thereof, nor any of their employees, makes any warranty, express or implied, or assumes any legal liability or responsibility for the accuracy, completeness, or usefulness of any information, apparatus, product, or process disclosed, or represents that its use would not infringe privately owned rights. Reference herein to any specific commercial product, process, or service by trade name, trademark, manufacturer, or otherwise does not necessarily constitute or imply its endorsement, recommendation, or favoring by the United States Government or any agency thereof. The views and opinions of authors expressed herein do not necessarily state or reflect those of the United States Government or any agency thereof. 